\title{
IMPACTO DE LOS EVENTOS DE SEQUÍA EN LA REGIÓN DE LA CUENCA HIDROGRÁFICA DE LA LAGUNA SAUCE GRANDE (PROVINCIA DE BUENOS AIRES, ARGENTINA)
}

\author{
María E. Carbone $e^{1,2}$, Claudia F. Fornerón ${ }^{1}$ y María C. Piccolo ${ }^{1,2}$ \\ ${ }^{1}$ Instituto Argentino de Oceanografía \\ ${ }^{2}$ Departamento de Geografía y Turismo
}

\section{RESUMEN}

La distribución e intensidad de los extremos hídricos provoca un impacto directo sobre las actividades humanas. Las características particulares de los eventos secos y húmedos ocurridos en la región de la cuenca hidrográfica de la laguna Sauce Grande, durante el período 1971- 2010, se analizan mediante la aplicación del Îndice de severidad de sequía de Palmer. El 49\% de los casos analizados correspondieron a sequías débiles, e incipientes. Los valores más extremos de eventos secos (-4.31) ocasionaron daños irreparables en los rendimientos de granos, que disminuyeron un 19\% respecto al promedio actual. Durante los meses estivales se observaron los casos secos severos y extremos (12\%). Se identificaron los períodos secos que mayor injerencia tuvieron sobre la producción agrícola ganadera de la región, sucedidas durante los años 2008 y 2009, la relación existente entre los valores de los índices severos y extremos en la morfometría de la laguna y estuario del río Sauce Grande.

Palabras clave: sequía, ISSP, morfometría, laguna Sauce Grande

\section{ABSTRACT}

\section{Drought impact in the region of the watershed the Sauce Grande lagoon} (Buenos Aires province, Argentina)

The distribution and intensity of extreme water causes a direct impact on human activities. Dry and wet events in the region of the watershed of the Sauce Grande lagoon during the period 1971 - 2010 are analyzed through the application of Index of Palmer drought severity, $49 \%$ of the cases analyzed corresponded to weak, and emerging drought. The most extreme dry events values (-4.31) caused irreparable damage yields, which decreased by $19 \%$ compared to the current average. During the summer months the severe and extreme (12\%) cases were observed. Dry periods had greater influence on livestock farming in the region, occurring during the years 2008 and 2009, the relationship between this values of the indices and severe extremes in the morphometry of the lagoon and the Sauce Grande estuary were identified.

Keywords: drougth, PSDI, morphometry, Sauce Grande lagoons

\section{INTRODUCCIÓN}

El déficit pluviométrico prolongado ocasiona un desequilibrio hidrológico que impacta directamente en el desarrollo de las actividades humanas (Hunt et al., 2009). La sequía no debe ser analizada como un simple fenómeno natural sino como el resultado de la interrelación de variables físicas, ambientales y

Contacto: María E. Carbone: ecarbone@criba.edu.ar; Claudia F. Fornerón: cforneron@gmail.com; María C. Piccolo: piccolo@criba.edu.ar

1 Camino La Carrindanga km 7. Bahía Blanca

2 UNS. 12 de Octubre y San Juan $4^{\circ}$ Piso. Bahía Blanca. 
antrópicas (Velasco et al., 2005).Los factores que agravan la severidad de la sequía como son las temperaturas altas, los vientos fuertes, humedad relativa baja, escasa humedad del suelo y prácticas territoriales inadecuadas que se realizan en los suelos productivos.

La sequía hidrológica es la permanencia del déficit hídrico en una región y altera el balance de agua. La sequía agrícola se manifiesta cuando la cantidad de precipitación, su distribución, las reservas de agua en el suelo y las pérdidas producidas por la evapotranspiración se combinan para causar una disminución considerable de los rendimientos del cultivo y el ganado (WMO,1975, FAO 1990 Barros et al., 2000). Una sequía está caracterizada por su intensidad, frecuencia, magnitud y dispersión geográfica. La identificación y el análisis de la intensidad de la sequía resultan de suma importancia para la prevención de las eventualidades que estas acarrean con respecto a la planificación de la economía de una región (Duran 1998, Alessandro 2008, Barros 2008). Es por ello que se considera de utilidad el seguimiento de las sequías en el tiempo y el espacio. Uno de los métodos más desarrollados para la detección y evaluación de las sequías es el Î́ndice de Severidad de Sequía de Palmer (Palmer, 1965).

Las sequías afectan, en forma continua a las regiones destinadas a la agricultura y ganadería en Argentina, incidiendo también en la morfometría de los cuerpos de agua que drenan en esas áreas. La ocurrencia de un año lluvioso o seco en el centro de este país, donde se localiza la llanura pampeana, se asocia a anomalías de la dinámica de los sistemas atmosféricos sobre las grandes cuencas oceánicas así como a la conexión con anomalías interanuales de la circulación atmosférica tales como el fenómeno ENSO (Vargas et al., 1999; Grim et al., 2000).

La región pampeana representa un 87 \% de la superficie cultivada con cereales y oleaginosas concentrando así la mayor parte de la producción de granos del país (INDEC, 2008). En esta zona se encuentra la cuenca hidrográfica de la laguna Sauce Grande, donde predominan los usos del suelo agrícola ganadero. Los cultivos de granos (soja, maíz, trigo y girasol) ocupan el $45 \%$ de la superficie total $\left(2500 \mathrm{~km}^{2}\right)$ y los forrajes el 39\%. La laguna Sauce Grande se considera como un centro de esparcimiento y recreación. La lámina de agua cuyo principal afluente es el río homónimo está habilitado para la pesca deportiva, embarcada y de costa (Fornerón, 2012).

La evaluación de la intensidad de las sequías en la región ha sido objeto de numerosos trabajos, principalmente el referido a la distribución espacio temporal de las mismas y el impacto directo sobre la producción agrícola (Valiente 2001, Andrade et al., 2009). Algunos autores identificaron las sequías del siglo XX para la región pampeana y su influencia sobre los rindes del cultivo de trigo (Scian y Donnari ,1997, Carbone et al., 2004, Piccolo et al., 2002), otros han analizado la influencia de la sequía sobre la producción de maíz (Minetti et al., 2007) La evaluación de esos eventos de déficit hídrico se centra principalmente en las campañas productivas de los últimos 50 decenios del siglo, advirtiéndose una correlación directa entre la sequía y el descenso del rendimiento de cereales (Carbone et al., 2008 , Scarpatti et al., 2011). Otros autores analizaron la disminución de la superficie cubierta por agua con respecto a períodos húmedos y normales del Dique Paso de las Piedras que provee de agua potable a núcleos urbanos del sur de la provincia de Buenos Aires (Marini, 2013, D’Ambrosio et al., 2013).

El objetivo de este trabajo de investigación es determinar los eventos de sequías en la región de la cuenca de la laguna Sauce Grande provincia de Buenos Aires, mediante la aplicación del ISSP. El período de estudio comprende los años 1971- 2010. Se analiza la influencia de los índices negativos en los rendimientos de cultivos de la región y en la variación de la morfometría de la laguna y estuario del río Sauce Grande.

\section{MATERIALES Y MÉTODOS}

Los eventos de sequías en la región se identifican a partir de la aplicación del Índice de Severidad de Sequía de Palmer (ISSP) con la utilización de un software específico (Ravelo, 1990). Fue creado por Palmer en 1965, quien clasificó los distintos estadios de humedad y sequías de acuerdo a las categorías presentadas en la Tabla 1. Los valores positivos indican exceso de humedad con respecto a los valores normales o medios, mientras que los valores negativos muestran condiciones de déficit hídrico. El indicador se calcula con datos termopluviométricos y con la disponibilidad de agua en el suelo. A partir de los datos de precipitación y evapotranspiración se pueden estimar todos los términos básicos de la ecuación del balance de agua, la recarga del suelo, la escorrentía y la pérdida de humedad del horizonte superficial. 
Tabla 1. Rangos de periodos húmedos y secos según Palmer (1965).

\begin{tabular}{|l|l|}
\hline \multicolumn{1}{|c|}{$\mathbf{X}$} & \multicolumn{1}{c|}{ Clases } \\
\hline$>4,0$ & Humedad extrema \\
3,0 a 3,9 & Humedad severa \\
2,0 a 2,9 & Humedad moderada \\
1,0 a 1,9 & Ligeramente húmedo \\
0,5 a 0,9 & Humedad incipiente \\
$-0,4$ a 0,4 & Normal \\
$-0,5$ a $-0,9$ & Sequía incipiente \\
$-1,0$ a $-1,9$ & Sequia reducida \\
$-2,0$ a $-2,9$ & Sequia moderada \\
$-3,0$ a $-3,9$ & Sequía severa \\
$<-4,0$ & Sequía extrema \\
\hline
\end{tabular}

Las variables que utiliza este indicador son: la temperatura mensual del aire $\left({ }^{\circ} \mathrm{C}\right)$, la precipitación mensual (mm) y el Contenido de Agua Disponible (CAD) del suelo (mm). Se determinó el balance hidrológico y el índice de anomalía de humedad (Ravelo, 1990). El modelo de balance hídrico utilizado considera dos capas de suelo: la capa superior (Ss) contiene $25 \mathrm{~mm}$ de agua útil y la capa inferior (Su) que contiene una determinada cantidad de agua útil en función de la profundidad considerada y las características propias del suelo.

Los registros pluviométricos mensuales utilizados correspondieron al período 1971 - 2010 para las estaciones meteorológicas de Coronel Dorrego, Coronel Suárez, Tres Arroyos, Bahía Blanca y Bordenave (Figura 1). Debido a la falta de datos meteorológicos de la estación de Laprida solo se analizó el período 1971 - 1990. Los datos pluviométricos y meteorológicos fueron obtenidos del Servicio Meteorológico Nacional (SMN), el Instituto Nacional de Tecnología Agropecuaria (INTA) y la Red de Información Agroeconómica de la Región Pampeana (INTA RIAP). Los mapas temáticos de los eventos de sequía se obtuvieron a partir de un Sistema de Información Geográfica (Arc Gis 10).

Figura 1. Localización de las estaciones meteorológicas en la región de la cuenca hidrográfica de la Laguna Sauce Grande.

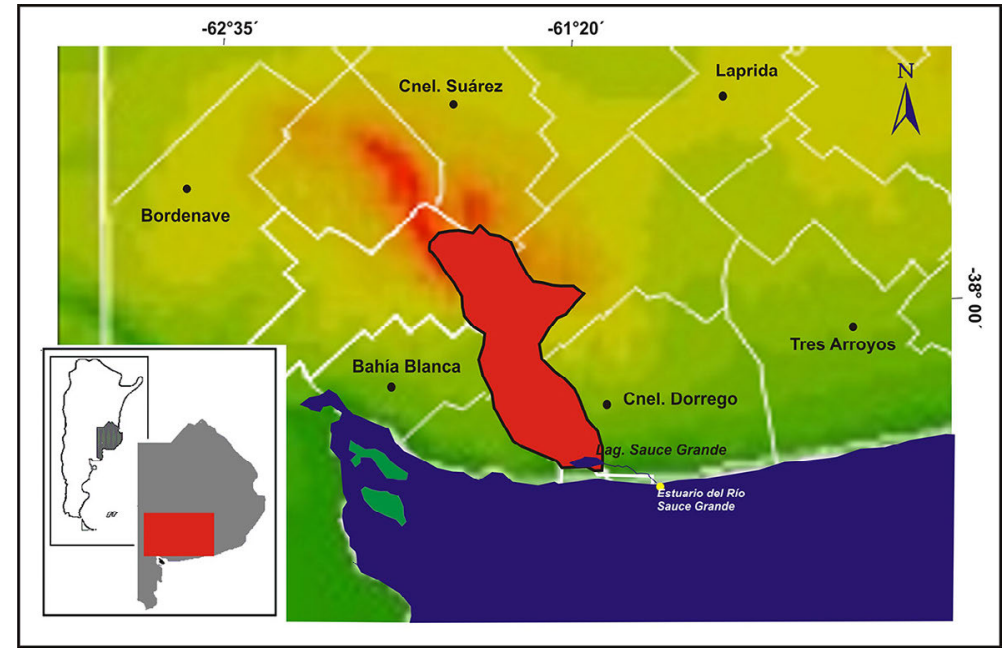

Con respecto al CAD para el área de estudio, se consideró una capacidad de almacenaje del suelo de $95 \mathrm{~mm}$ (Coronel Dorrego), $101 \mathrm{~mm}$ (Coronel Suárez), $103 \mathrm{~mm}$ (Tres Arroyos), 96 (Bahía Blanca), 100 (Bordenave) y $104 \mathrm{~mm}$ (Laprida). Estos datos fueron cedidos por el Instituto Nacional de Tecnología Agropecuaria (INTA). La evapotranspiración fue calculada mediante la fórmula de Penman - Monteith (FAO 1990)

En el balance hidrológico se estimó la evapotranspiración (ET), la recarga (R), el escurrimiento (Ro) y pérdida de humedad de suelo (L). Además se determina: $\alpha$ o Coeficiente de Evapotranspiración (ET / PE), ß o Coeficiente de Recarga (R / PR), $\gamma$ o Coeficiente de Escurrimiento (RO / PRO), $\delta$ o Coeficiente de Pérdidas (L / PL). Utilizando estos coeficientes se determinó la cantidad de precipitación climática o normal para cada mes de acuerdo a la ecuación de balance superficial de agua (Palmer,1965): 


$$
\hat{P}=E \hat{t}+\hat{R}+R \hat{o}+\hat{L}
$$

La diferencia entre la precipitación actual y la precipitación necesaria para mantener el promedio de humedad climática o normal Palmer (1965) la define como apartamiento de humedad (d).

$$
d=p-\hat{p}
$$

Durante los períodos húmedos estos apartamientos son positivos y durante los secos son negativos. Además se obtuvo el índice de anomalías de humedad (z) multiplicando los valores d por una constante $\mathrm{k}$ determinada para cada lugar y para cada período $(z=d . k)$. La constante $\mathrm{k}$ se estimó como la relación demanda - suministro de humedad y se la representó según:

$$
k=\overline{E T P}+\bar{R} / \bar{P}+\bar{L}
$$

\subsection{Calculo del índice de sequía}

Se calculó el índice de sequía X, para lo que se consideraron los períodos más secos (extremos) para distintos intervalos de tiempo. Siguiendo la metodología propuesta por Palmer (1965) se obtuvo la recta que representa la tasa de secado máxima ocurrido para diferentes períodos del fenómeno (meses), involucrando los extremos de todas las localidades y considerando como categoría de sequía extrema el valor de -4 . Se calculó la ecuación de la recta a través de:

$$
\underset{\substack{I \\ X_{I}=\sum}}{I} Z_{i} /(a . t+b)
$$

Los milímetros de anomalías de humedad $\left(\mathrm{Z}_{\mathrm{i}}=\mathrm{k}_{\mathrm{j}} \mathrm{d}\right)$ se transformaron a índices mensuales de sequía y se normalizaron, para determinar el factor de peso climático regional k a través de la siguiente ecuación:

$$
\hat{k}_{j}=1.5 \log _{10}\left(T_{j}+2.8\right) / \overline{D_{j}}+0.50
$$

Se calculó el ISSP a través de la siguiente ecuación:

$$
\Delta x_{i}=z_{i} /(a . t+b)+c x_{i-1}
$$

Considerando que el índice de un mes está influenciado por el estado de humedad del mes anterior se calcula el coeficiente c (Donnari y Scian, 1993) de la siguiente ecuación:

$$
x_{i}-x_{i-1}=\left(z_{i} / 40.09\right)+c x_{i-1}
$$

El impacto de la sequía en la región se determinó a través de la incidencia de los índices negativos en los rindes de cultivos de las diferentes localidades. Además se analizó el impacto de los eventos secos y húmedos sobre la morfometría de la laguna Sauce Grande. Se utilizaron imágenes satelitales Landsat 5 TM, 7 ETM y Spot (Convenio CONAE IADO).

\section{RESULTADOS Y DISCUSIÓN}

\subsection{Variabilidad del ISSP}

Los datos correspondientes a los años 1971 - 1980 indican que los períodos secos predominaron sobre los húmedos en el área analizada. Las sequías débiles y moderadas con un ISSP de hasta -2,06 se observaron durante los meses de enero y marzo de 1971. Para el siguiente año entre enero y mayo la sequía de cinco meses obtuvo valores de sequía incipiente hasta moderada con un ISSP de -2,39. Para el período de agosto 1975 y febrero 1976 la sequía de siete meses alcanzó la categoría moderada con valores de hasta $-2,78$. El evento seco de máxima duración temporal y de mayor intensidad, se detectó entre marzo de 1977 y mayo de 1978. El mismo duró quince meses con categorías de sequías incipientes a severas con valores de hasta -3,39. El último evento seco del período, se observó entre diciembre de 1978 y abril de 1979 con una duración de cinco meses, con categorías de sequía incipientes y débiles (Figura 2a). 
Durante el decenio 1981 - 1990 se manifestaron cuatro eventos secos. Entre junio y noviembre de 1981se detectó el evento seco de menor intensidad del período con un valor del ISSP de -1,52. Entre junio y agosto de 1988, se observaron tres meses de sequía débil con un ISSP de -2,32. En el período entre noviembre de 1988 y abril de 1989, se detectó el evento seco de mayor intensidad del período con un valor del ISSP de -3,01. El mismo duró seis meses donde se identificaron sequías severas (Figura 2b).

Para el período 1991 - 2000 se manifestaron siete eventos secos. Entre marzo y octubre de 1990 se observaron 8 meses con déficit hídrico, de los cuales 3 se clasificaron como sequía incipiente y 5 como sequía débil con un valor de ISSP que alcanzó los -1,63. La sequía más intensa y duradera del período con nueve meses secos se observó entre mayo de 1995 y enero de 1996, con una calificación de severa alcanzando un valor del ISSP de hasta -3,23. De junio a octubre de 1996 siguieron los eventos secos con un valor de índice de -1,72. El último evento seco detectado se extendió entre noviembre de 1998 y marzo de 1999 con 5 meses secos, donde 2 fueron de sequía incipiente, 1 de sequía débil, 1 de sequía moderada y 1 de sequía severa con un valor máximo del ISSP de -3,02 (Figura 2c).

Para el primer decenio del siglo XXI (2001 - 2010) se observaron hasta mediados del periodo eventos secos que varían entre sequía moderada y débil (ISSP -2.85). Entre noviembre 2007 y junio 2009, se observó el evento extremo más seco de la década y del período analizado. Presentó una duración de diecisiete meses, de los cuales los primeros ocho variaron entre sequía débil y moderada. En los últimos meses los eventos de déficit hídrico manifestaron categoría severa a extrema con valores ISSP de hasta -4,31. Entre noviembre de 2009 y marzo de 2010 los meses secos alcanzaron índices de severidad de -3,75 (Figura 2d).

Figura 2. Marcha temporal del ISSP en la región para el período correspondiente a 1971-2010.

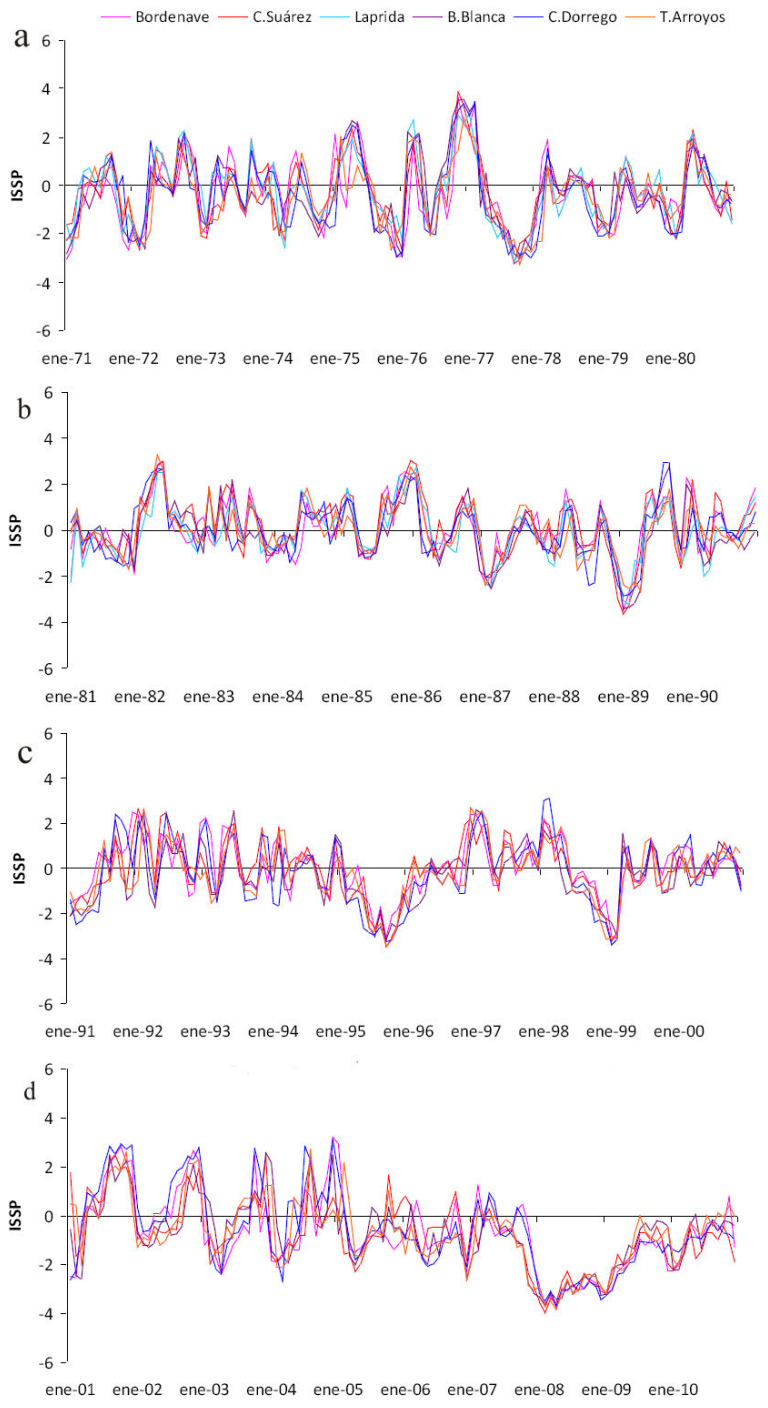




\subsection{Análisis de la frecuencia y duración de los eventos hídricos}

Los casos de déficit hídricos analizados en las cuatro décadas (1971-2010) son superiores a los de humedad en la región (49\%). Se analizaron 480 episodios para cada una de las localidades. Los casos de rango normal representan el 28,2\%. Se observó un 35\% de sequía débil e incipiente (o reducidas), la sequía moderada representó un $9 \%$, la sequía severa $4 \%$ y los casos extremos representan el $2 \%$ del total estudiado. Los valores de índice positivo representan el $21,2 \%$, donde la mitad corresponde a casos de humedad incipiente, el $6 \%$ de humedad moderada, el $4 \%$ severa y exceso hídrico alcanza el 1,5\% del record estudiado (Figura 3).

Figura 3. Frecuencia de eventos secos y húmedos en el área de estudio.

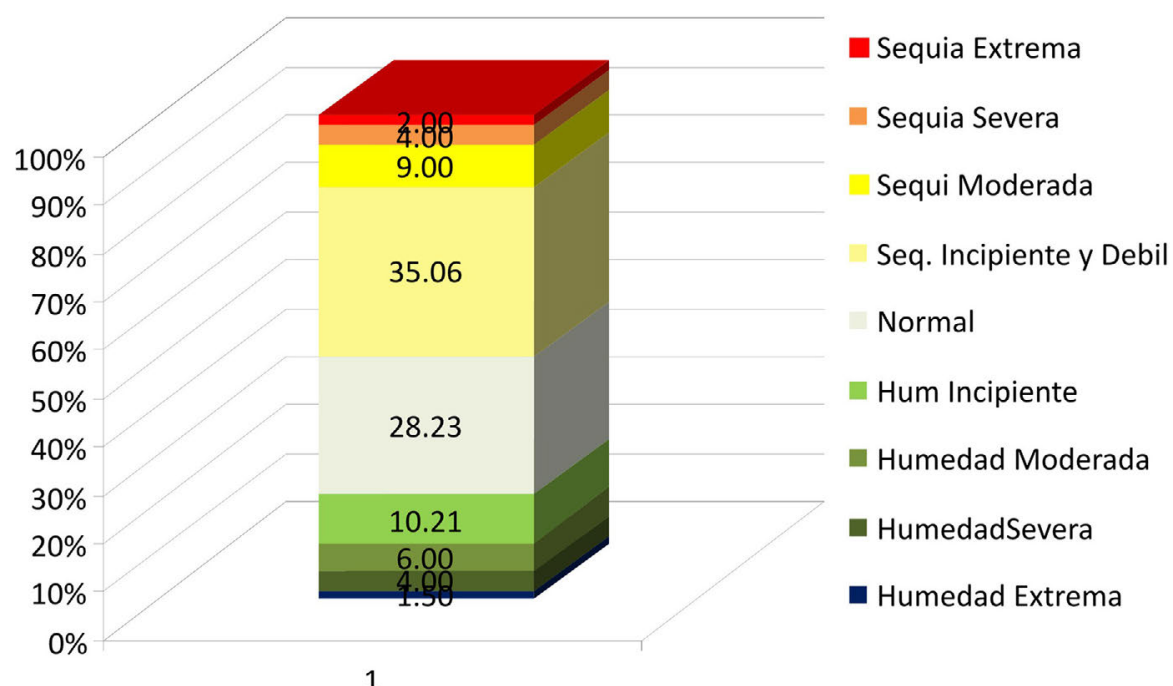

Los mayores porcentajes de sequías a escala mensual (Figura 4) corresponden al rango débil e incipiente. El mes que mayor porcentaje presenta de esta última categoría es junio coincidente con el periodo de siembra de trigo. Los eventos de sequía moderada se presentan en el primer y último trimestre del año, alcanzando el máximo porcentaje en abril (28,9\%). La sequía severa y extrema representan el $6 \%$ del total de casos analizados, siendo los meses estivales donde se presentan frecuentemente. Durante el mes de febrero se acentúa el déficit hídrico con un $12,5 \%$ de eventos secos extremos

Figura 4. Distribución mensual de rachas secas.

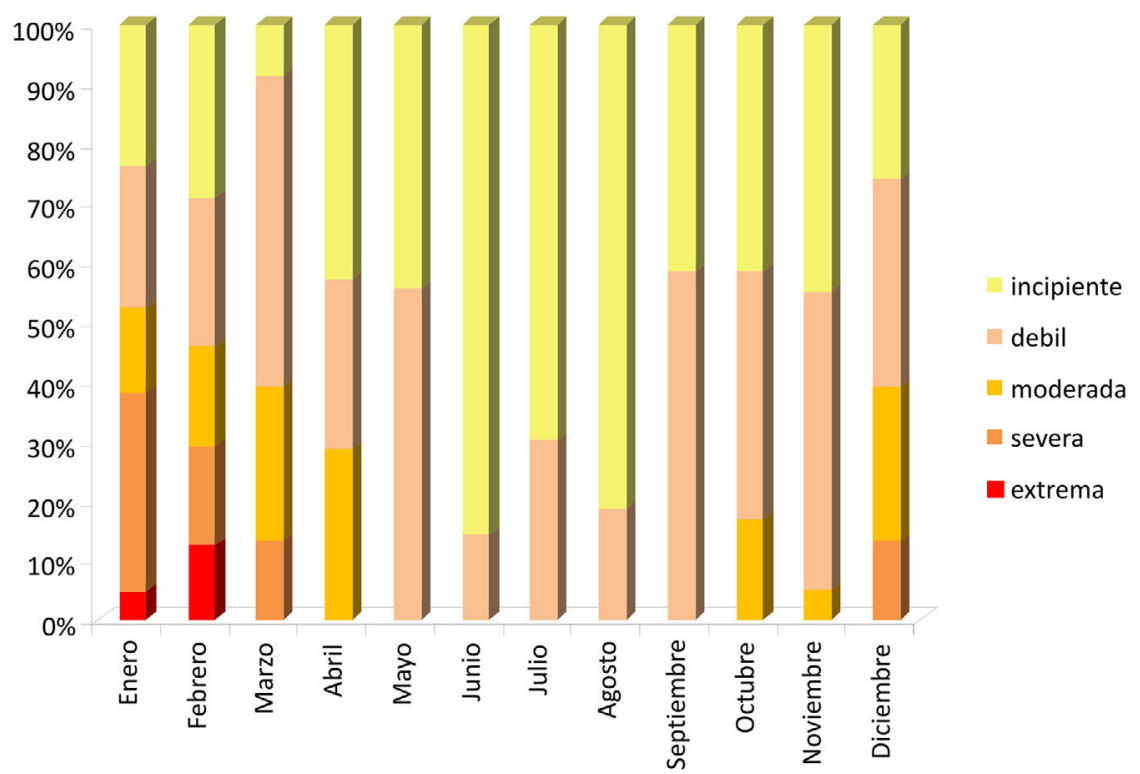




\subsection{Distribución espacial e intensidad de dos casos de sequía edáfica}

El primer episodio se extiende desde abril a agosto de 2008 y el segundo desde diciembre de 2008 hasta febrero de 2009. En abril del año 2008 se evidencian dos núcleos de sequía severa en la región Un núcleo se observa instalado en la localidad de Bahía Blanca y el otro hacia el norte cercano a la localidad de Coronel Suárez. Estos núcleos poseen valores de ISSP de -3,15 y -3,05, respectivamente. En el centro de la región y en la cuenca hidrográfica de la laguna Sauce Grande, se observa una situación de sequía moderada con valores de ISSP que variaron entre -2,51 y -1,98 (Figura 5).

Hacia mayo de 2008, se observa que los núcleos de sequía severa se extendieron y formaron uno en la parte central de la región, desde la localidad de Bordenave hasta Coronel Dorrego abarcando la parte norte de la cuenca de la laguna. Los valores del índice aumentaron a -3,26. Rodeando este núcleo y extendiéndose desde el centro al sur de la cuenca hidrográfica de la laguna se observa una situación de sequía moderada con valores de hasta $-2,65$. Los valores de ISSP son más altos respecto a los del mes anterior. En junio de 2008, el núcleo de sequía severa del mes anterior se desplaza hacia el sur de la región cubriendo la localidad de Bahía Blanca, Coronel Dorrego, Tres Arroyos y la cuenca hidrográfica de la laguna, excepto la parte norte. Los valores del ISSP varían entre -3,32 y -3,50. En Coronel Suárez se observa una situación de eventos moderados con valores de hasta $-2,70$ cuyo núcleo se debilita hacia la localidad de Bordenave. En julio de 2008, el núcleo de sequía severa se desplaza hacia el sur, cubriendo por completo las localidades de Bahía Blanca, Coronel Dorrego, Tres Arroyos y la cuenca hidrográfica de la laguna. Los valores alcanzaron -3,78 evidenciándose situaciones de déficit hídrico más severo, El resto de la región presentó situación de sequía moderada con índices que varían entre -2,75 y -2,89.

Figura 5. Distribución espacial de la sequía (Abril - Agosto 2008) en la región de la cuenca hidrográfica de la laguna Sauce Grande (Fornerón, 2013).
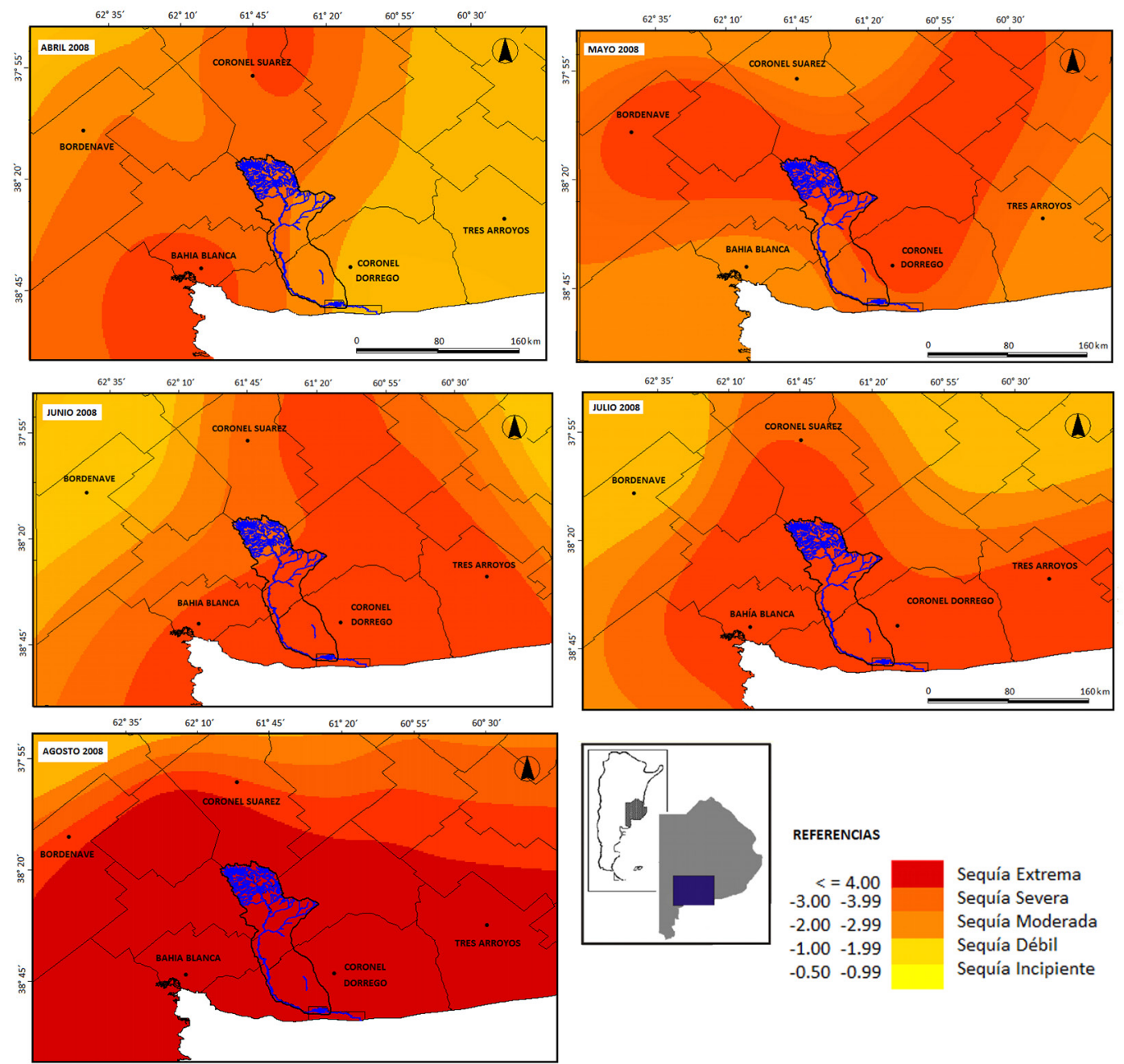
En agosto de 2008, se evidencia un núcleo de sequía extrema que afecta a todo el sur de la región, incluyendo la cuenca de la laguna Sauce Grande. El mismo presenta valores del ISSP de -4,31. Esta racha seca se agrava por poseer meses acumulados de índices negativos severos y extremos.

El segundo episodio analizado se presenta en la región desde diciembre de 2008 a marzo 2009 (Figura 6). La sequía se presenta con un rango severo durante el primer mes y se extiende desde la localidad de Bordenave hasta Coronel Dorrego, incluyendo el sector norte y medio de la cuenca hidrográfica de la laguna Sauce Grande. Los valores del ISSP oscilaron entre -3,4 y -3,8. En el sector este de la región predominó una situación de sequía moderada con un ISSP de -2,7. Al norte, el núcleo se debilita y Coronel Suárez presenta un valor del ISSP de -2,1. En enero de 2009, el núcleo de sequía severa del mes anterior se convierte en sequía extrema cubriendo la zona desde Bahía Blanca a Coronel Suárez, atravesando el sector medio de la cuenca hidrográfica de la laguna. Los valores del ISSP alcanzan -4,2. En febrero de 2009, el núcleo de sequía extrema desaparece y las condiciones de sequía severa se extienden desde la localidad de Bahía Blanca hasta Tres Arroyos, incluyendo la mayor parte de la cuenca hidrográfica de la laguna. Los valores del ISSP variaron entre -3,4 y -3,6. La sequía se debilita hacia el oeste y noroeste de la región y se presentan situaciones de sequía moderada con valores del ISSP de -2,7 y -2,1.

Figura 6. Distribución espacial de la sequía (Diciembre 2008 - Febrero 2009) en la cuenca hidrográfica de la laguna (Fornerón, 2013)
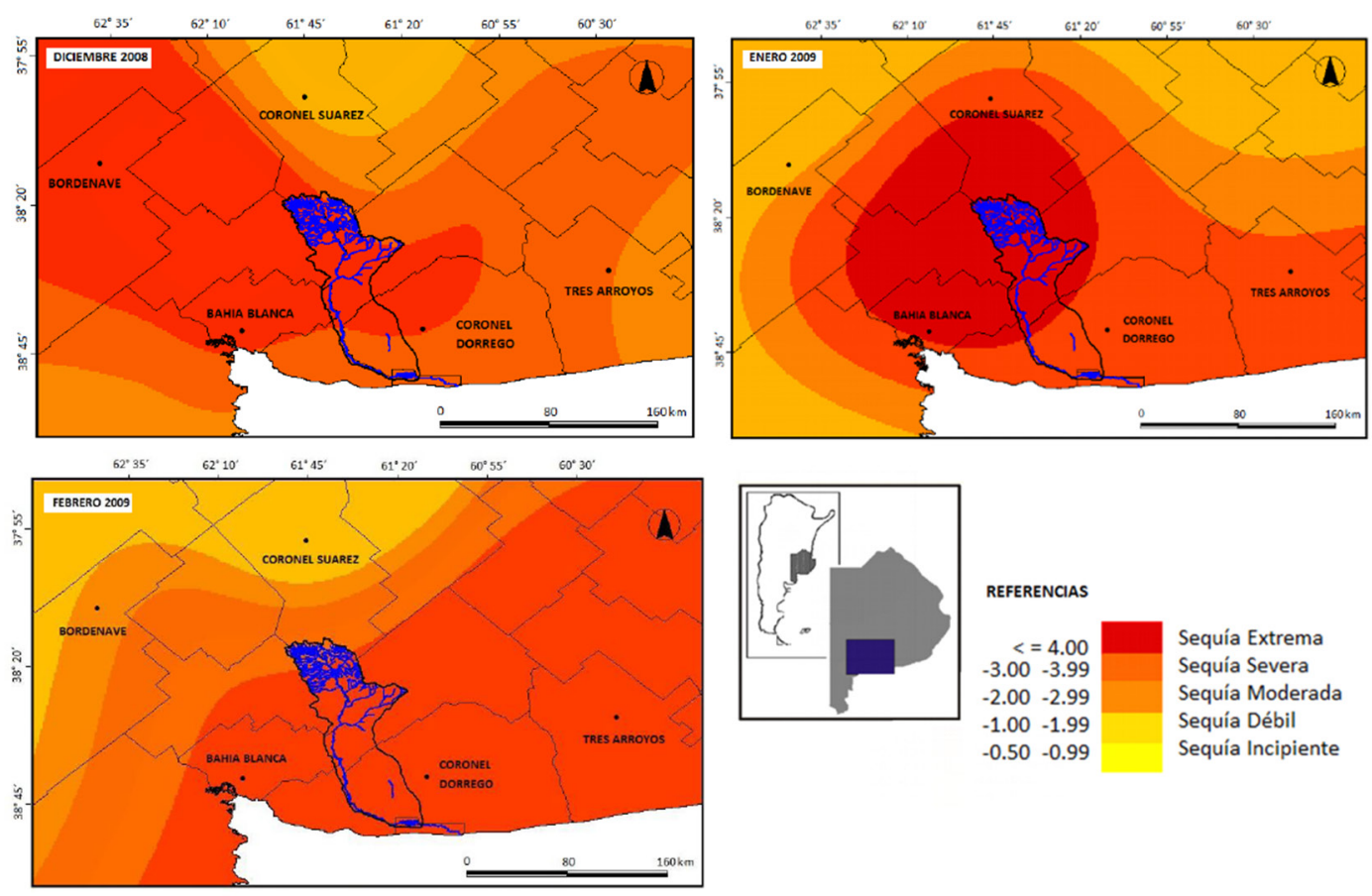

\subsection{Impacto de la sequía en la región de la cuenca hidrográfica de la laguna Sauce Grande}

Para poder determinar los impactos de esa sequía en los cultivos de la cuenca hidrográfica se han analizado las campañas agrícolas correspondientes al periodo 2004 - 2010. Durante el año 2008 y 2009 ocurre un nuevo episodio de "La Niña" que afecta toda la Región Pampeana. En esta ocasión este fenómeno se presenta con anomalías negativas de temperatura y precipitaciones, estas ultimas con valores de -167 a $-200 \mathrm{~mm}$ (Sierra, 2012). Los rindes productivos fueron afectados por estas condiciones dado que el déficit de humedad en la columna del suelo complica la siembra y posterior desarrollo de los cultivos como el trigo.

Para relacionar el impacto de los periodos de sequía sobre los rendimientos de los cultivos de la región se realiza el análisis sobre uno de los cultivos de mayor producción como es el trigo en el área de estudio. En la figura 7 se presenta la relación directa que existe entre la presencia de ISS negativo asociado a una disminución de la producción triguera. Los valores negativos indican la presencia de un período de sequía. Se aprecia además rendimientos por debajo de los valores de la media (3250 kg/tn) para las campañas agrícola analizadas. 
Figura 7. Comparación entre el ISS y el rinde promedio de trigo para el período 2004 - 2010

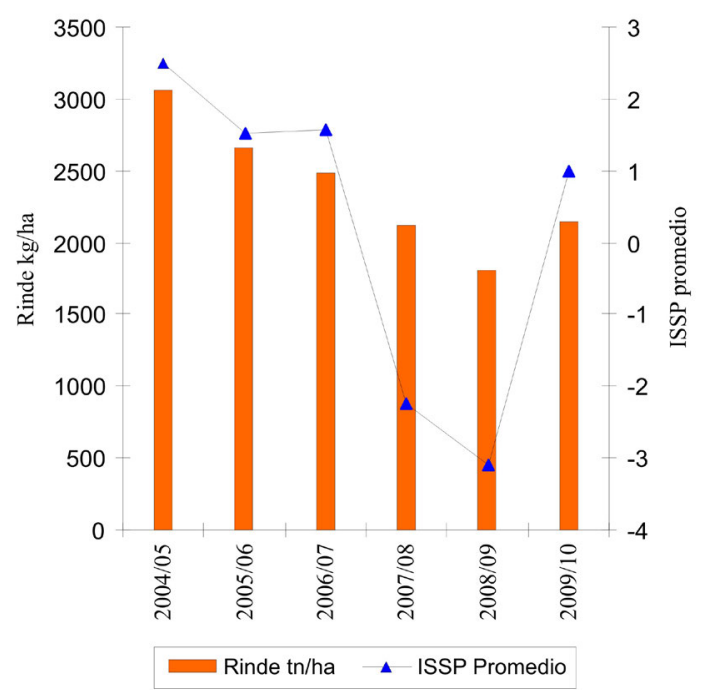

Durante las campañas agrícolas de cereal en la región correspondientes a los años 2007/08 y 2008/09 los rindes disminuyeron entre un 17 y 19\%. Se registraron valores de $2.122 \mathrm{~kg} / \mathrm{ha}$ y $1.805 \mathrm{~kg} / \mathrm{ha}$ respectivamente, muy inferior a la media de los rindes actuales $(3.022 \mathrm{~kg} / \mathrm{ha})$. La gran sequía ocurrida desde abril a septiembre 2008 afectó el sudeste bonaerense y disminuyó notoriamente los rendimientos de trigo, girasol, maíz y soja (Coma, 2010). El bajo rendimiento de las pasturas obligó a completar con granos la alimentación de la hacienda aumentando los costos de producción. La caída de los rendimientos marcó el impacto negativo de esta sequía. En algunos campos de Coronel Pringles y Coronel Dorrego se instalaron equipos de riego en zonas donde los acuíferos contaban con suficiente caudal, pero no fue suficiente para tratar de disminuir el daño provocado por la deficiencia hídrica. Además del déficit hídrico este sector de la llanura pampeana soportó más de quince días de heladas severas.

\subsection{Incidencia de los eventos hídricos en la morfometría de dos cuerpos de agua de la región de estudio}

Las variación espacial de la laguna Sauce Grande y del estuario del río homónimo se analizó teniendo en cuenta los años que se indican en la Tabla 2 considerados los más representativos de los extremos hídricos. En el año 1987 (año con valores de ISSP normal) el área de la laguna era de 19,6 km². Durante el año 1990, la laguna presentó un área de 20,2 $\mathrm{km}^{2}$ incrementándose en un 7\% transcurriendo el año 1991 donde el ISSP indica un año de Humedad Severa. En 1995 en presencia de eventos de sequía extrema disminuye la superficie lagunar un 8\%. En el año 1998 (Normal) la laguna disminuyó su superficie a 21 km².

En el año 2002 la superficie lagunar aumentó a $23,5 \mathrm{~km}^{2}$ durante este periodo ocurre una de los excesos hídricos más importantes de principio de siglo XXI para esta región. En el año 2004 la laguna alcanza una superficie de $23 \mathrm{~km}^{2}$. En el año 2008, categorizado como de Sequía Extrema, el área lagunar fue de $17,2 \mathrm{~km}^{2}$ lo que representa una reducción del área del $26 \%$ en relación al 2002. Se evidencia que la laguna Sauce Grande en las épocas más húmedas se expande hacia el sector Norte y Oeste coincidente con la localización de las zonas anegadas del ecosistema.

Tabla 2. Clasificación de los años analizados para la variación areal de la laguna Sauce Grande y Estuario del río homónimo

\begin{tabular}{|cc|}
\hline Clases & Años \\
\hline Humedad Incipiente & 1991 \\
Humedad severa & $2002-2004$ \\
Normales & $1987-1990-1998$ \\
Sequía Extrema & $1995-2008$ \\
Sequía Moderada & 2010 \\
\hline
\end{tabular}


Durante el año 2010 este cuerpo de agua redujo su superficie a 18 km². Entre 2004 y 2010 el área lagunar disminuyó un 21 \% (Figura 8). La disminución de la superficie lagunar afecta directamente la vida acuática de este ecosistema. La escasa profundidad que presenta este cuerpo lagunar, durante las rachas secas (prof. media $1.60 \mathrm{~m}$, mínima $0.89 \mathrm{~m}$ ), puede generar condiciones nocivas para la fauna ictícola que allí habita, dado que se alteran todos los parámetros físico químicos del agua.

Figura 8. Variación areal de la laguna Sauce Grande para el período 1987 - 2010 (Fornerón, 2013).

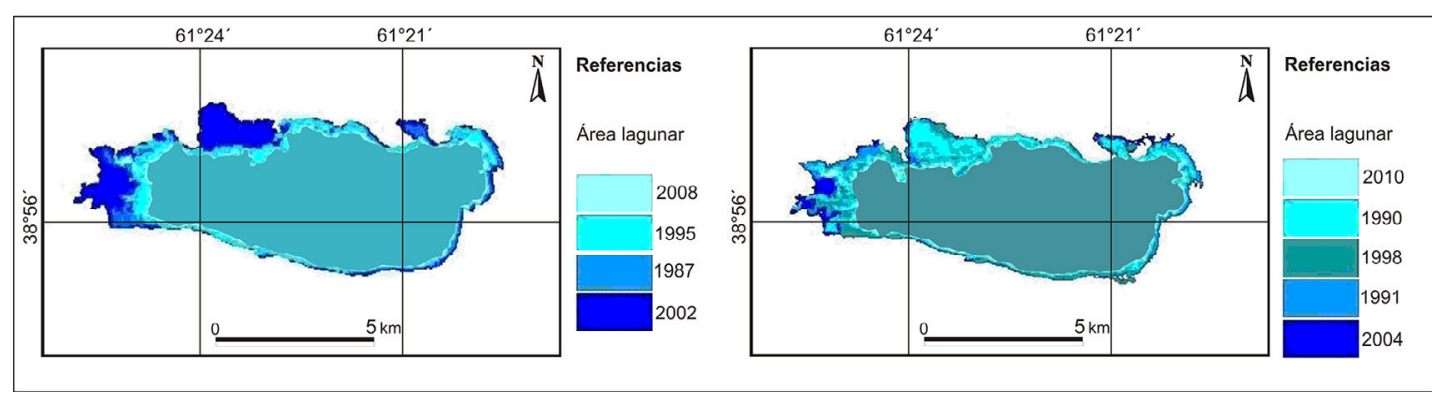

La figura 9 presenta la variación areal y espacial del estuario del río Sauce Grande. El tamaño de este ecosistema durante los años clasificados como normales $(1987,1998)$ de acuerdo a los valores de ISSP alcanzaba dimensiones de hasta $0,30 \mathrm{~km}^{2}$. Durante el año 1990 el área de la desembocadura del río era de $0,31 \mathrm{~km}^{2}$ aumentando su extensión un $16 \%$ en 1991. La superficie estuarial disminuyó a $0,20 \mathrm{~km}^{2}$ en 1995 durante un episodio de sequía extrema. En el año 2002 (Humedad Severa) la superficie aumentó a $0,39 \mathrm{~km}^{2}$. En el año 2004 el estuario presentó una disminución del área a $0,34 \mathrm{~km}^{2}$, si bien este año también es considerado con humedad severa durante el 2002 la humedad en la columna de suelo era superior, debido a precipitaciones acumuladas que totalizaron $158 \mathrm{~mm}$ más de los valores normales $(710 \mathrm{~mm})$.

Figura 9. Variación areal del estuario del río Sauce Grande entre los años 1997 y 2010 (Fornerón, 2013).

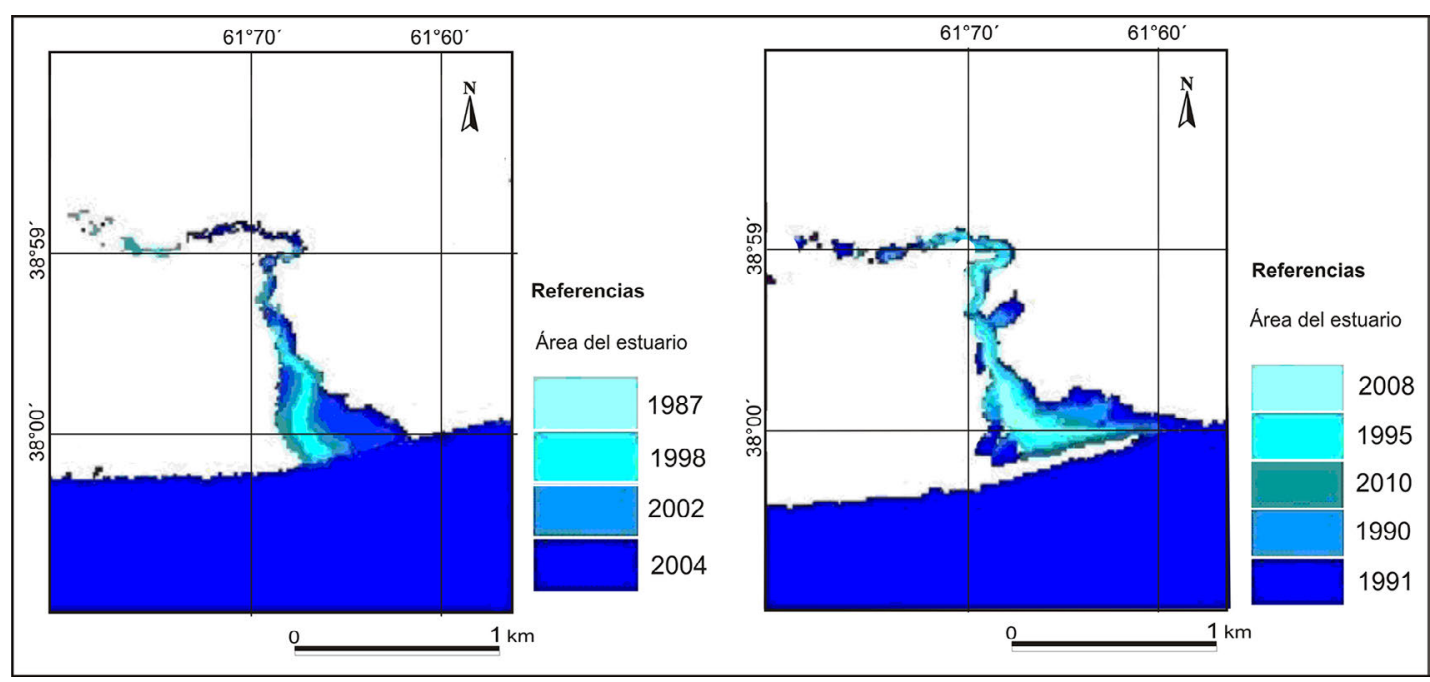

Cuando los valores de ISSP resultaron negativos y extremos (2008) el área del estuario del río disminuyo hasta llegar a $0,17 \mathrm{~km}^{2}$. Cuando la condición de racha seca alcanzó a ser severa, el cuerpo de agua incrementó su superficie a $0,25 \mathrm{~km}^{2}$. La dinámica espacial del estuario se evidencia en cada uno de los cambios producidos durante los diferentes eventos analizados. El río presentó dos bocas diferenciadas: la principal, correspondiente al sector actual (Figura 9b) y otra menor perteneciente al antiguo curso (Figura 9a). Esta última boca sólo se activa durante los períodos lluviosos (Fornerón et al., 2009; Fornerón et al., 2010c).

\section{CONCLUSIONES}

La aplicación del Índice de Severidad de Sequía de Palmer permitió caracterizar la distribución e intensidad de sequía en la región de la cuenca de la laguna Sauce Grande. En todo el período analizado 
predominaron las situaciones de sequía incipiente y débil en un $30 \%$ y $48 \%$, respectivamente. Se lograron detectar períodos de sequías prolongados y severos.

El episodio seco más importante tuvo lugar entre los años 2008 - 2009, coincidente con lo ocurrido en toda el área que ocupa la llanura pampeana. Los valores que indican sequías extrema alcanzaron índices de hasta -4.30. Esta situación de déficit hídrico produjo un alto impacto en la actividad agrícola ganadera. Las consecuencias directas fueron reducción de la producción cerealera, mortandad de hacienda y peces. Además esta situación fue agravada por las anomalías negativas de precipitaciones que desde julio a agosto de 2008 alcanzaron valores de hasta $-200 \mathrm{~mm}$.

La dinámica espacial y temporal del estuario del río y de la laguna Sauce Grande tuvo relación con el ISSP de la región. Ante un ciclo de sequía o humedad el área de estos cuerpos de agua se modificó. El análisis integrado de los índices y la variación areal permite obtener información válida para elaborar pautas de manejo de estos ecosistemas,

La aplicación de un índice con variables integradas como sucede con el ISSP permite contar con una herramienta muy útil para la gestión y planificación en eventos de extremos hídricos. El diagnóstico que se obtiene con el conocimiento de la frecuencia, distribución e intensidad de los eventos secos y húmedos se traduce en la elaboración de mapas temáticos de seguimiento de los núcleos de estos eventos, que pueden ser utilizados para predecir situaciones similares y recurrente en áreas productivas como es la región pampeana argentina.

\section{AGRADECIMIENTOS}

Este trabajo fue financiado parcialmente por subsidios del CONICET, proyecto redes PAMPA 2, Universidad Nacional del Sur y el Interamerican Institute for Global Change Research (IAI) CRN 3038 (under US NSF award GEO-1128040).

\section{RECURSOS ELECTRÓNICOS}

ALESSANDRO A.P. (2008): Anomalías de circulación atmosférica en 500 y 1000 hpa asociada a la sequía producida en la Argentina durante enero de 2003 a marzo de 2004, Revista Brasileira de Meteorologia, v.23, n.1, pp. 12-29,

ANDRADE, M.I., LAPORTA, P. y IEZZI, L. (2009): Sequías en el sudoeste bonaerense: Vulnerabilidad e incertidumbre [En línea].Geograficando, 5 (5).

BARROS, V. A. (2008): Adaptation to climatic trends: lessons from the argentine experience. pp. 296-350. In: N. Leary, I. Burton, J. Adejuwon, V. Barros, R. Lasco and J.i Kulkarni (eds). Climate change and adaptation. Earthscan, London.

BARROS, V., GONZÁLEZ, M., LIEBMANN, B. \& CAMILLONI, I. (2000): Influence of the South Atlantic convergence zone and South Atlantic sea surface temperature on interannual summer rainfall variability in South-eastern South America. Theoretical and Applied Climatology, 3(67), 123-133.

CARBONE, M.E. y PICCOLO, M.C. (2004): "Análisis de los períodos secos y húmedos en la cuenca del Arroyo Claromecó, Argentina". Papeles de Geografía, 40, pp. 25-36.

CARBONE, M.E., SCIAN, B. and PÍCCOLO, M.C. (2008): Agricultural drought in the Claromeco river basin, Buenos Aires province, Argentina Revista de Climatologia. Vol 8, pag. 113, Agencia Estatal de Meteorología. España

COMA, C. (2010): Trigo en el área de la Estación Experimental de INTA Bordenave Antecedentes de producción y actualidad. RIAN RIAP

D’AMBROSIO, G.T., BOHN V.Y., PICCOLO, M.C. (2013): Evaluación de la sequía 2008-2009 en el oeste de la Región Pampeana (Argentina). Cuadernos Geográficos 52 (1), pp. 29-45

DONNARI, M. y SCIAN, B. (1993): Sequías Edáficas en Bordenave, Método de Palmer Revista Geofísica, vol. 39, pp. 85-99.

DURÁN, D. (1998): Las sequías como riesgo natural. En La Argentina ambiental. Naturaleza y Sociedad. Buenos Aires, Lugar Editorial. pp. 71-138.

FAO (1990): Crop water requeriments. Irrigation and drainage, Paper 24, 144 p.

FORNERÓN, C.F., PICCOLO, M.C. y CARBONE, M.E. (2009): Variación morfológica del estuario del río Sauce Grande. VII Jornadas Ciencias del Mar, Instituto Argentino de Oceanografía (IADO) Bahía Blanca. 
FORNERÓN, C.F., PICCOLO, M.C., CONY, N., y FERRER, N. (2010): Hidrografía de la laguna Sauce Grande durante el otoño de 2010 (Provincia De Buenos Aires). Contribuciones Científicas Sociedad Argentina de Estudios Geográficos - Volumen 22, pp. 197-206.

FORNERÓN, C.F. (2013): Hidrografía de la laguna Sauce Grande (provincia de Buenos Aires) en época de sequía. Tesis Doctoral .UNS, $216 \mathrm{p}$.

FORTE LAY, J., SCARPATI, O.E. \& CAPRIOLO, A.D. (2008): Precipitation variability and soil water content in pampean flatlands (Argentina). Geofisica Internacional, 47(4), pp. 341-354. http://scielo. unam.mx/scielo.

GRIMM, A.M., BARROS, V.R., DOYLE, M.E. (2000): Climate variability in southern South America associated with El Niño and La Niña event, J. Clim, 13(1), pp. 35-58.

HUNT, E.D., HUBBARD, K.G., WILHITE, D.A., ARKEBAUER, T.J. \& DUTCHER, A.L. (2009): The development and evaluation of a soil moisture index. Int. J. Climatol., 29(5), pp. 747-759. Revista Brasileira de Meteorologia, v.23, n.1, pp. 12-29.

MARINI F., (2009) Sequía en el área de influencia de la EEA Bordenave. Evolución del índice verde durante 2009. Informe INTA Bordenave.

MISTERIO DE AGRICULTURA, GANADERÍA Y PESCA DE LA NACIÓN MAGyP (2011): Herramientas para la evaluación y gestión del riesgo climático en el sector agropecuario Argentina. Coord. Occhiuzzi S., Mercurui P y Pascale C. $1^{\circ}$ Ed. Bs. As. 130p.

MINETTI, J.L., VARGAS,W. M., VEGA, B. y COSTA, M.C. (2007): Las sequías en la Pampa Húmeda: impacto en la productividad del maíz. Revista Brasileira de Meteorologia, v.22, n.2, pp. 218-232.

PALMER, W.C. (1965): Meteorological drought. U.S. Weather Bureau, Washington D.C. Research Paper No $45,58 \mathrm{p}$.

PICCOLO. M.C., CAPELLI DE STEFFENS, A. y CAMPO DE FERRERAS, A. (2002): "La sequía del año 1995 en el sur de la región pampeana argentina." Desastres Naturales en América Latina, J. Lugo y M. Inbar (Eds), pp. 189-206.

RAVELO, A. (1990): Manual Teórico Operativo del Índice de Sequía de Palmer.

SCARPATI, O., FORTE LAY, J. A. y CAPRIOLO, A. (2011): Drought risk in argentine pampean region. International Journal of Ecology and Development, 18, pp. 1-18. Retrieved from http://ceser.in/ceserp/ index.php/ijed/article /view/871

SCIAN, B. and DONNARI, M. (1997): Aplicación del índice Z de Palmer para la comparación de sequías en las regiones trigueras II, IV y V Sur de Argentina. Revista de la Facultad de Agronomía-UBA, 17(I), pp. 41-46.

SIERRA, E. (2012): Perspectiva agroclimática para el final de la campaña agrícola 2011/2012 y el comienzo de la Campaña agrícola 2012/2013. Agrositio Bolsa de Cereales Buenos Aires 15p.

MARCOS VALIENTE, O. (2001): Sequía: definiciones, tipologías y métodos de cuantificación. Investigaciones Geográficas, 26, pp. 59-80

VARGAS, W.M., PENALBA, O., MINETTI, J. (1999): Las precipitaciones mensuales de la Argentina y el ENOS. Un enfoque hacia el problema de la decisión. Meteorológica 24, pp. 3-18.

VELASCO, I., OCHOA, L. y GUTIÉRREZ, C. (2005): Sequía, un problema de perspectiva y gestión. En:Región y Sociedad, Vol XVII, No 34, 2005, Sonora, El Colegio de Sonora, México. http://www.maa. gba.gov.ar/agricultura-est.

WMO. (1975): World Climate Programme. Data and Monitoring. Drought and Agriculture.Technical Note No 138. WMO. 